Running head: assessment of sexual orientation-related OCD

\title{
Assessing sexual orientation-related obsessions and compulsions in Italian heterosexual individuals: Development and validation of the Sexual Orientation Obsessive-Compulsive Scale (SO-OCS)
}

Gabriele Melli ${ }^{1,2}$, Richard Moulding ${ }^{3}$, Simona Gelli $^{2}$, Carlo Chiorri ${ }^{4}$ and Antonio Pinto ${ }^{5}$

\section{1) University of Pisa}

2) Institute for Behavioral and Cognitive Psychology and Psychotherapy of Florence (IPSICO)

3) Centre for Mental Health and Wellbeing Research, Deakin University, Melbourne, Australia

4) University of Genova

5) Cognitive and Behavioral Psychotherapy Center, Naples, Italy

Correspondence to:

Gabriele Melli, PsyD

Via Mannelli, 139 - 50132 Firenze - Italy

Email address: g.melli@ipsico.it

Telephone: +390552466460

Fax: +39055 2008414 


\begin{abstract}
Sexual Orientation - Obsessive-Compulsive Disorder (SO-OCD) is characterized by intrusive thoughts, images and urges related to one's sexual orientation, and by consequent avoidance, reassurance seeking, and overt and covert compulsions. Currently there is no short selfreport measure that assesses SO-OCD symptoms. The current article describes two studies that develop and evaluate the first version of the Sexual Orientation Obsessive-Compulsive Scale (SOOCS) - a 14-item Italian self-report measure targeted towards heterosexual individuals. In Study 1, the SO-OCS was developed and refined through item analysis and exploratory factor analysis from an initial pool of 33 items administered to 732 Italian non-clinical participants. The SO-OCS showed a unidimensional structure and an acceptable internal consistency. In Study 2, the factor structure, internal consistency, temporal stability, construct and criterion validity, and diagnostic sensitivity of the SO-OCS were investigated in three samples of Italian participants (294 from the general population, 52 OCD patients who reported sexual orientation-related symptoms or concerns as a primary complaint, and 51 OCD patients who did not report these symptoms as primary complaint). The SO-OCS was again found to have a unidimensional structure and good internal consistency, as well as to exhibit strong construct validity. Specifically, the SO-OCS showed an excellent criterion validity and diagnostic sensitivity, as it successfully discriminated between those with SO-OCD and all other groups of participants. Finally, evidence of temporal stability of the SOOCS in a non-clinical subsample was found. The SO-OCS holds promise as a measure of SO-OCD symptoms in heterosexual individuals.
\end{abstract}

Keywords: Obsessive-compulsive disorder; sexual orientation; obsessions; assessment 
Assessing sexual orientation-related obsessions and compulsions in Italian heterosexual individuals:

Development and validation of the Sexual Orientation Obsessive-Compulsive Scale (SO-OCS)

\section{$\underline{\text { Introduction }}$}

Obsessive compulsive disorder (OCD) is one of the most common mental disorders with a lifetime prevalence of 1.9-2.5\% across cultures (Nedeljkovic, Moulding, Foroughi, Kyrios, \& Doron, 2012; Weissman et al., 1994), and it is also one of the ten most debilitating medical conditions according to the World Health Organization (Murray \& Lopez, 1996). It is characterized by the occurrence of persistent thoughts, urges, or images that are experienced as intrusive and unwanted (obsessions), and compulsive actions that the individual feels driven to perform in response to an obsession, which are aimed at preventing or reducing anxiety or distress, or preventing some dreaded event or situation from occurring (American Psychiatric Association [APA], 2013). Excluding hoarding, which is now recognized as reflecting a separate disorder (APA, 2013), typical manifestations of OCD include contamination/cleaning; ordering/symmetry, and unacceptable/taboo thoughts (Bloch, Landeros-Weisenberger, Rosario, Pittenger, \& Leckman, 2008) with doubt/checking occurring across these symptom domains.

Sexual obsessions are prominent within the unacceptable thoughts symptom dimension with the most common themes of sexual obsessions concerning unfaithfulness, pedophilia, incest, AIDS, profane thoughts combining religion and sex, and obsessions concerning one's sexual orientation (Cathey \& Wetterneck, 2013). Concerns regarding one's sexual orientation are prominent in this subtype of obsessions - given that sexuality is a topic often laden with moral, emotional, and religious meaning, it is clearly a fertile topic for obsessions (Gordon, 2002).

Williams (2008) defined "homosexual OCD" as "the obsessive fear of being or becoming homosexual, the experience of intrusive, unwanted mental images of homosexual behavior, and/or the obsessive fear that others may believe one is homosexual" (p.197). It can also manifest as the inability to tolerate doubt itself about one's sexual orientation. While generally seen with respect to fears of one having a gay sexual orientation ${ }^{1}$, it is possible for there to be unwanted anxiety 
regarding unwanted heterosexual thoughts in individuals who are lesbian/gay/bisexual/transgender (L.G.B.T.; see for a case example, Goldberg, 1984); as such homosexual OCD is now more generally known as sexual orientation OCD (SO-OCD; Williams, Tellawi, Davis, \& Slimowicz, 2015; Williams, Wetterneck, Tellawi, \& Duque, 2014).

This subtype of OCD is characterized by obsessive rumination on the meaning of one's intrusive and unpleasant thoughts and images with regards to sexual orientation. As with rituals related to other types of repugnant obsessions (see Moulding, Aardema, \& O'Connor, 2014), mental rituals in SO-OCD may include a large number of cognitive acts such as mental repetition of special words, mental reviewing, and mental acts to reverse what has been done (mental undoing). More specific compulsions including repeating expressions - for example, in people with a heterosexual sexual orientation: "I don't like people of the same sex"; "My family and my friends know that I don't like people of the same sex"; or "Everyone knows that I fall in love with people of the opposite sex". A heterosexual person with SO-OCD may also try to visualize two persons of the same sex kissing, and check that there is a negative visceral reaction to this image in order to confirm that she/he is not becoming gay. Some heterosexual SO-OCD patients might review past experiences with the opposite sex to try to convince themselves that they have never gained pleasure from such situations. They may further avoid situations that provoke the unwanted thoughts, such as locker rooms, movies that have same-sex themes, and sometimes even people perceived to be members of the L.G.B.T. community (Williams et al., 2014). Similarly, they may worry about a sudden lack of attraction to people of the opposite sex. As such, they may engage in testing behaviors, such as by have intercourse with their partner or by masturbating while watching pornography, in order to ensure that they are "still straight" (Williams, 2008). This form of checking is particularly counterproductive, as the OCD-related anxiety may result in a decreased sex drive and/or in performance anxiety, which the heterosexual individual then further misinterprets as evidence that they are gay (Williams, 2008). Finally, people with SO-OCD sometimes ask other people for reassurance, but as it is generally the case with reassurance in OCD, 
any sense of relief is brief and the doubts always return (Williams, 2008). These individuals may also use other forms of reassurance including self-reassurance, the need to confess to others, and compulsively searching the internet. However, excessive demands for reassurance can contribute to family distress (Williams, Crozier, et al., 2011). Even though a professional may diagnose them with OCD, until they are successfully treated individuals with SO-OCD often doubt the diagnosis. As noted by Williams et al. (2014) SO-OCD is differentiated from those who are actually conflicted about their sexuality (Gordon, 2002), from homophobia (Williams, 2008), and also from internalized homophobia, which is when a L.G.B.T. person has negative feelings about him/herself on account of their sexual orientation (Szymanski, Kashubeck-West, \& Meyer, 2008).

Using data collected as part of the OCD Collaborative Genetics Study (Samuels et al., 2006), Pinto et al. (2008) reported that $9.9 \%$ of their sample $(n=485)$ endorsed past or present obsessions related to fears of their being gay. Williams and Farris (2011) directly examined the prevalence and the characteristics of individuals reporting obsessions concerning their sexualorientation, in a large clinical sample originally from the DSM-IV field trial $(n=409)$, using the Yale-Brown Obsessive-Compulsive Symptom Checklist and Severity Scale (Y-BOCS). The authors found that $16.8 \%$ reported current sexual obsessions and $9.5 \%$ past obsessions, with $8 \%(n=33)$ specifically reporting current sexual-orientation obsessions and $11.9 \%(n=49)$ endorsing lifetime symptoms. Males were more likely to experience this kind of obsessions. These patients appeared to have similar insight to people with other types of OCD but experienced higher levels of distress, interference, and avoidance, and spent more time on obsessions, when compared with other subtypes of OCD. Thus, SO-OCD may be more distressing than are other forms of OCD, indicating a pressing need for research on its assessment and treatment.

Unfortunately, until recently no self-report measures for OCD were available that allowed a specific evaluation of the sexual-orientation OCD symptoms. In particular, sexual orientation concerns were only addressed on the main clinical OCD measure (the Y-BOCS checklist) by a single item that asks if obsessional content involves (or involved) being gay. Addressing this, 
Wetterneck, Siev, Adams, Slimowicz, and Smith (2015) developed an adaptation of the

Dimensional Obsessive-Compulsive Scale (DOCS) that assesses Sexually Intrusive Thoughts as a further dimension; however this symptom dimension is relatively broad and therefore lacks utility for specifically assessing SO-OCD. More specifically addressing SO-OCD, Williams et al. (2014) developed a survey comprising 70 novel items that explored worries about and fears of becoming or being L.G.B.T., worries that others may think one is L.G.B.T., and concerns regarding experiencing unwanted same sex thoughts, which they examined using a large online sample. The purpose of the study was to better understand sexual orientation worries and the distress related to such intrusive thoughts. The SO-OCD survey questions were found to load upon six subscales: worry about a change in sexual orientation, desire for same sex partners, desire for other sex partners, beliefs that a same-sex orientation is wrong or immoral, avoidance of other's judgment, and sexual orientation shame. Alarmingly, among people with SO-OCD, 91\% had levels of distress ranging from "much" to "suicidal" as a result of their sexual orientation obsessions, confirming the strong importance of developing assessment scales and treatment options for this presentation of OCD. The main limitation of this study concerned the generalizability of the sample, which comprised online participants and therefore may only have been representative of those with a particular interest in that topic. While the study did include individuals with OCD, this clinical information was based on self-report that individuals had received a prior diagnosis. Overall, while the research by Williams et al. (2014) is both important and novel in its revelations about SO-OCD, the length of their instrument preclude its routine use in clinical and research settings which generally require a rapid, tailored and effective assessment.

The current study, which was begun independently of the recent studies by Williams et al. (2014) and Wetterneck et al. (2015), was prompted by the need for a specific assessment measure of SO-OCD (in comparison to Wetterneck et al., 2015), which is short (in comparison to Williams et al., 2014), has robust psychometric properties, and which holds utility for clinical and research settings. A measure of sexual orientation fears would allow for relevant further investigations of 
SO-OCD phenomenology and the relationship of SO-OCD to other OCD symptoms and to other disorders, as well as for research into its prevalence and potential predictors. However, such a measure also requires affinity for a clinical setting in order to facilitate appropriate recognition and assessment of SO-OCD. For example, in clinical settings, we would envisage that such a measure might be useful in the instance where sexual orientation obsessions are possible but have not been noted by a client (i.e., it is sometimes easier to answer honestly to standard questions on a questionnaire than in an interview with a new clinician). Equally, such a measure may be useful to track symptom severity during therapy for those with identified SO-OCD, or when a clinic is undertaking a comprehensive OCD assessment. Indeed, many clinics are incorporating measures in the move to evidence-based practice, so for specialist OCD clinics or for clinicians who are referred many OCD (or anxiety) clients it could form a standard part of such a battery. This would counteract the current state of affairs where we believe that this OCD subtype is often misunderstood and misdiagnosed, and as such the individual's access to potential treatments is limited.

In sum, this study aimed to develop a new scale for clinical and research settings, which we have denoted as the Sexual Orientation Obsessive-Compulsive Scale (SO-OCS). Given that the majority of individuals with SO-OCD are heterosexual patients with concerns over being gay, the first version of the scale, which is presented in the current paper, was targeted towards heterosexual individuals (SO-OCS-V1). Please note that we have also devised a version of the scale whose content reflects heterosexual-orientation obsessions-related concerns for use with L.G.B.T. populations (SO-OCS-V2), which we are independently evaluating. In this paper, for clarity, we use the SO-OCS acronym with reference to SO-OCS-V1.

\section{Study 1}

Study 1 was geared towards initial development of the SO-OCS scale, examining an initial pool of draft items within a large community sample. The aim was to develop an adequate and 
psychometrically sound measure of sexual orientation obsessions, which could then be further examined in clinical populations.

\section{$\underline{\text { Method }}$}

\section{Participants}

The sample consisted of 732 (66.9\% Female) heterosexual community volunteers $(M=$ 35.76 years; $S D=13.27$; range $18-70$ ) who responded to advertisements requesting potential volunteers for psychological studies in Central Italy. In terms of education, $62.5 \%$ of the participants had a medium level of education (12-13 years, high school degree), $25.8 \%$ had a higher-level degree (16 or more years, bachelor's degree or Ph.D.) and the remaining $11.7 \%$ had a low level of education (8 or less years, primary or secondary school). Most were employed (58.4\%), $31.7 \%$ were undergraduate university students, and the remaining $9.9 \%$ were homemakers, unemployed, or retired. Regarding marital status, $47.5 \%$ were single, while $47.4 \%$ were married or cohabiting, $3.7 \%$ were divorced, and $1.4 \%$ were widows or widowers.

\section{Measures}

Sexual Orientation Obsessive-Compulsive Scale (SO-OCS). A preliminary version of the SO-OCS was designed according to recommendations for scale development (Furr, 2011), and consisted of 33 items, written in Italian, generated by the authors of this paper on the basis of their expert knowledge and practical experience of assessment and treatment of OCD. Seventeen of the items were worded to assess specific obsessions - e.g., "I often worry about suddenly discovering that I am homosexual"; the remaining sixteen were worded to assess compensatory behaviors - e.g., "I feel the need to repeatedly check if I am attracted to men or women", or "I check myself, physically and mentally, to ensure that I am not sexually aroused when I happen to see an attractive same-sex person". These initial items were then sent to a group of experts on OCD and psychometricians not otherwise involved in the study; they were asked to evaluate the relevance and representativeness of the draft items to the construct and to suggest amendments that would improve the content and face validity of the items. Some individuals with SO-OCD symptoms 
provided feedback on the readability, comprehensibility and relevance of the items. Following the feedback, 12 items were removed and others were amended to improve clarity, specificity and relevance. The final SO-OCS consisted of twenty-one items. No reverse-scored items were included. Instructions read as follows: "This questionnaire has been developed only for those who have always had a wholly or mainly heterosexual sexual orientation. In other words, it is for those who are mainly attracted to opposite-sex people. There is no reason to fill out this questionnaire if you instead have a predominantly homosexual orientation. Please read the statements below and assess with the utmost honesty how much you agree with each one of them, considering the last month and using the following reference scale: $0=$ Not at all; $1=$ A little; $2=$ Moderately; $3=\mathrm{A}$ lot; 4 = Extremely.”

\section{Procedure}

All participants volunteered to take part in the study after being presented with a detailed description of the procedure, and signed a written informed consent and were treated in accordance with the Ethical Principles of Psychologists and Code of Conduct (American Psychological Association, 2010). The questionnaire took less than 5 minutes to be completed. No external incentives were offered for participating in this study.

\section{Statistical analysis}

Exploratory factor analysis (EFA) and item analysis were conducted to identify items for possible elimination due to weak psychometric properties. Following this item deletion procedure, EFA and item analysis were performed again to examine the factor structure and the reliability of the final solution. The number of factors to extract was determined according to the Scree-plot, the Minimum Average Partial correlation statistic (MAP; Velicer, 1976), and parallel analysis (PA) with optimal implementation (Timmerman \& Lorenzo-Seva, 2011). On the basis of the recommendations of Buja and Eyuboglu (1992), PA was performed on 1000 random correlation matrices obtained through permutation of the raw data, and following Longman, Cota, Holden, and 
Fekken (1989), both the mean eigenvalues and the 95th percentile eigenvalues were considered. These analyses were performed with FACTOR8 (Lorenzo-Seva \& Ferrando, 2006).

Since a substantial number of items showed values of skewness and kurtosis that fell outside the $[-1 ;+1]$ range recommended by Muthén and Kaplan (1985) for using maximum likelihood estimator, factor analyses were then performed in Mplus 6.1 using the mean and variance adjusted weighted least squares estimator (WLSMV; Muthén, Du Toit, \& Spisic, 1997). When using WLSMV estimator, Mplus 6.1 provides fit indices for EFA analogous to those of Confirmatory Factor Analysis (CFA), i.e., the Tucker-Lewis Index (TLI), the Comparative Fit Index (CFI), and the Root Mean Square Error of Approximation (RMSEA). Following Marsh, Hau, and Wen (2004), values $\geq .90$ were considered as acceptable and $\geq .95$ as optimal for the TLI and the CFI, and values $\leq .08$ as acceptable and $\leq .06$ as optimal for the RMSEA. The use of multiple indices provides a conservative and reliable evaluation of model fit when compared to the use of a single fit index.

\section{$\underline{\text { Results }}$}

The Kaiser-Meyer-Olkin (KMO, Kaiser, 1974) measure of the sampling adequacy was .92, and Bartlett's test of sphericity was significant, suggesting that the correlation matrix was suitable for factor analysis. Examination of the Scree-plot suggested that eigenvalues markedly began to level off after one factor (first five observed eigenvalues: 14.31, 1.09, .71, .65, .51), PA suggested the extraction of only one factor both when mean percentile was considered and when 95th percentile was considered, and MAP reached its lowest value at one factor $(.022, .027, .039, .057$, $.140)$. Taken together, these results suggested that the one factor solution was the most appropriate, and EFA was performed with the number of factors to extract set to 1 .

Although both the goodness-of-fit indices $(\mathrm{RSMEA}=.04 ; \mathrm{CFI}=.98 ; \mathrm{TLI}=.97)$ for the onefactor solution and also internal consistency $(\alpha=.86)$ were very good, our aim was to create a measure with an adequate factor structure and sound psychometric properties but that is still short enough for clinical and research application. In addition, the goal was to include a similar number of item assessing obsessions and neutralization behaviors. Hence, two items with the lowest factor 
loading $(<.65)$ and seven items with a corrected item-total correlation below .45 were removed. The final scale consisted of 14 items ( 7 for obsessions and 7 for neutralizing behaviors).

A second EFA was conducted on the remaining items and the one-factor solution showed excellent fit indices $(\mathrm{RMSEA}=.04 ; \mathrm{CFI}=.99, \mathrm{TLI}=.98)$, and all the items substantially (i.e., $\geq$ .79) loaded on the first factor, which accounted for $71 \%$ of the variance. Cronbach's alpha indicated that reliability was very good $(\alpha=.89)$. Corrected item-total correlations were all greater than .45 , and the mean inter-item correlation was .38 (range .22-.63). In no case was the alpha-if-item-deleted statistic higher than the computed alpha, suggesting that all items contributed to the internal consistency of the scales. Results of item and distribution analyses and of the EFA are shown in Table 1.

\section{Study 2}

In Study 1, we developed a measure of SO-OCD, that both had adequate psychometric properties and was short enough for routine clinical and research use. In Study 2 we sought to replicate these findings on an independent sample of non-clinical, heterosexual participants. We also examined whether the measure exhibited convergent validity with general obsessional symptoms as opposed to other OCD and non-OCD symptoms (anxiety, depression, worry) and a subsample of these participants also completed the SO-OCD twice at a 4-week interval to evaluate its test-retest reliability. Moreover, as clinical populations differ from non-clinical populations, we assessed the psychometric properties of the questionnaire within clinical samples.

\section{Method}

\section{Participants}

The total study sample consisted of 397 heterosexual adults, including 52 adults diagnosed with OCD who reported sexual orientation-related symptoms or concerns as a primary complaint (SO-OCD group), 51 adults who met the diagnostic criteria for primary OCD, but who did not report these symptoms or concerns as primary complaint (NSO-OCD group), and 294 non-clinical participants (NCP group) recruited from the general population independently of those of Study 1. 
Both participants in the SO-OCD group and the NSO-OCD group were referred to an Italian private center for adult psychotherapy for evaluation and treatment. They could not be included in the study if they were younger than 18 . The presence of psychosis, current mania, and/or substance dependence were exclusionary criteria. During the routine assessment phase clinical participants were interviewed by one of the members of our research team (all are doctoral-level psychologists experienced in diagnosing psychiatric disorders) using the Anxiety Disorder Interview Schedule-IV (ADIS-IV; Brown, Barlow, \& Di Nardo, 1994) and the Yale-Brown Obsessive-Compulsive ScaleSecond Edition (Y-BOCS-II; Melli, Avallone et al., 2015; Storch et al., 2010) to establish diagnoses. Each case was audio-recorded and carefully reviewed in supervisory meetings, and all diagnoses were confirmed by second-rater consensus (inter-rater reliabilities were excellent: ADIS$\mathrm{IV}=.96 ;$ Y-BOCS-II = .95). Comorbid disorders in the SO-OCD group included anxiety disorders (social phobia $[n=1]$, panic disorder $[n=2]$ and generalized anxiety disorder $[n=4]$ ) and mood disorders (major depressive disorder $[n=7]$ ). Comorbid disorders in the NSO-OCD group included anxiety disorders (social phobia $[n=1]$, panic disorder $[n=3]$ and generalized anxiety disorder $[n=$ 5]) and mood disorders (major depressive disorder $[n=11]$ ). Non-clinical participants lived in urban and suburban areas of Florence and responded to advertisements requesting potential volunteers for psychological studies. To be included in the study, they had to be at least 18 years old, have at least a primary school education, and report having never received a diagnosis or treatment for a psychiatric disorder. Socio-demographic information about the samples are reported in Table 2.

\section{Measures}

Sexual Orientation Obsessive-Compulsive Scale (SO-OCS). As described in Study 1. Obsessive-Compulsive Inventory-Revised (OCI-R; Foa et al., 2002). The OCI-R is a brief, 18-item, self-report questionnaire designed to measure obsessive-compulsive symptom-related distress. Each item is evaluated on a 5-point rating scale ranging from 0 ('not at all disturbed') to 4 ('extremely disturbed'). The OCI-R assesses symptoms on six different dimensions including 
washing, checking, ordering, obsessing, hoarding, and mental neutralizing (three items each). The Italian version of the OCI-R has been found to have good internal consistency $(\alpha=.85)$, excellent test-retest reliability $\left(r_{t t}=.93\right)$ for the total score, and adequate internal consistency $(\alpha \mathrm{s}=.60-.80)$ and test-retest reliability $\left(r_{t t} \mathrm{~s}=.76-.99\right)$ for each subscale (Sica et al., 2009). In the present study, all the subscales showed adequate internal consistency ( $\alpha$ s in the range $.61-.81$ across groups).

Penn State Worry Questionnaire (PSWQ; Meyer, Miller, Metzger, \& Borkovec, 1990). The PSWQ is a 16-item inventory designed to assess trait worry. Response choices are scored from 1 ('not at all typical of me') to 5 ('very typical of me'). The scale has shown good psychometric properties, and previous research indicates that the PSWQ can adequately distinguish worry from obsessions (e.g., Burns, Keortge, Formea, \& Sternberger, 1996). The Italian version of the PSWQ (Morani, Pricci, \& Sanavio, 1999) has been shown to have adequate internal consistency $(\alpha=.85)$ and construct validity. In the present study, internal consistency was also very good or excellent ( $\alpha$ s in the range $.88-.93$ across groups).

Beck Anxiety Inventory (BAI; Beck \& Steer, 1990). The BAI is a 21-item, self-report inventory that assesses the severity of anxiety. Response choices are scored from 0 ('not at all') to 3 ('severely'). The original version has shown good psychometric properties, and in a series of studies, the Italian version of the BAI has shown a one-factor structure, good internal consistency ( $\alpha$ $>.80$ ), adequate test-retest reliability $\left(r_{t t}>.62\right)$, and good construct validity (Sica, Coradeschi, Ghisi, \& Sanavio, 2006; Sica \& Ghisi, 2007). In the present study, internal consistency was also very good or excellent ( $\alpha$ s in the range .88-.94 across groups).

Beck Depression Inventory-II (BDI-II; Beck, Steer, \& Brown, 1996). The BDI-II is a 21-item self-report instrument that is used to assess depressive symptoms over the previous two weeks. Response choices are scored from 0 ('absent') to 3 ('severe'). The BDI-II has shown good psychometric properties, and the Italian version of the BDI-II (Sica \& Ghisi, 2007) has been shown to have a one-factor structure, adequate internal consistency ( $\alpha$ s in the range $.80-.87$ ), test-retest 
reliability $\left(r_{t t}=.76\right)$ and construct validity. In the present study, internal consistency was very good or excellent ( $\alpha$ s in the range .88-.93 across groups).

\section{Procedure}

The same procedure as in Study 1 was followed for this study. In addition, the measures were administered in a counterbalanced fashion to control for order and sequence effects. Batteries took between 15 and 25 minutes to be completed. One-hundred non-clinical participants completed the SO-OCS twice at a 4-week interval and their data were used to test the temporal stability of scores. No external incentives were offered for participating in this study.

\section{Statistical analysis}

A CFA was performed to replicate the results of Study 1 on the NCP group only, using the same method and criteria for evaluating goodness-of-fit. While it would be ideal to utilise the clinical population in this procedure, not surprisingly the distributions of item scores markedly differed between the SO-OCD and the NSO-OCD group, yielding substantially bimodal distributions when the two groups were combined. Combining different subgroups into one group can lead to biases in the estimation of the correlation matrix, on which factor analytic methods rely. As the OCD sample was not sufficiently large to perform a standalone CFA analysis as suggested by a power analysis performed following the suggestions of Muthén and Muthén (2002), data from the NCP group only was utilized. However, an EFA was performed on the clinical data as de Winter, Dodou, and Wieringa (2009) suggested that 50 cases can lead to reliable results when the expected factor loadings are in the $.80 \mathrm{~s}$ and a single latent factor is to be extracted. Given the distributions of item scores were skewed, the polychoric correlation matrix was used.

Internal consistency of the scale was calculated with Cronbach's alpha coefficients and distribution and item analyses were carried out in each group. Temporal stability was tested in the subsample of non-clinical participants by computing the correlation between scores of the first and the second administration and also by comparing mean scores with a paired-samples $t$-test. Construct validity of the SO-OCS was explored through inspecting the Pearson's correlation matrix, 
in both the OCD and NCP groups. The $Z_{\text {contrast }}$ test (Westen \& Rosenthal, 2003) was used to test whether the association of the SO-OCS with the OCI-R obsessing subscale (convergent measure) was significantly larger than those with the other subscales of the OCI-R and with other administered measures (discriminant measures). To test the criterion-related validity of the scale, a main-effect-only general linear model (GLM) was specified with the SO-OCS score as the criterion variable, group membership as the focal variable and demographic variables (age, gender, marital status, etc.) as control variables.

To ascertain the discriminative power of the SO-OCS, receiver operating characteristic curves (ROC; Metz, 1978; Swets, 1996) were computed. The ROC analysis uses the association between sensitivity and specificity to derive an area under the curve which indicates how well a measure distinguishes between case positive (i.e., SO-OCD patients) and case negative (i.e., NSOOCD patients or non-clinical controls) in a given sample, irrespective of the base rate. A value of 0.50 of the area under the curve indicates chance level and 1.0 indicates a perfect diagnostic tool. Finally, we established the cut-off scores with optimal diagnostic accuracy for distinguishing between individuals with SO-OCD and those with NSO-OCD, and between individuals with SOOCD and non-clinical participants.

\section{$\underline{\text { Results }}$}

\section{Factor analyses}

CFA was performed in the NCP sample $(n=294)$, and the one-factor model showed an excellent fit to the data $(\mathrm{CFI}=.98, \mathrm{TLI}=.98, \mathrm{RMSEA}=.05)$. EFAs performed on the data of the SO-OCD and the NSO-OCD subgroups showed that the one factor solution explained $48 \%$ and $74 \%$ of the variance, respectively, and all items substantially loaded on the single factor. Factor loadings ranged from .54 to .82 in the SO-OCD group and from .74 to .89 in the NSO-OCD group.

\section{Item analysis and reliability}

Table 3 displays the results of distribution and item analyses across the three groups. The minimum requirement for internal consistency was met (Kline, 1993); Cronbach's alpha showed 
very good reliability in all the groups ( $\alpha$ s in the range .85-.90). Corrected item-total correlations ranged from .33 to .43 . In no case was the alpha-if-item-deleted higher than the computed alpha, suggesting that all items contributed to the internal consistency of the scale.

As stated earlier, one-hundred non-clinical participants completed the measure a second time after a 4-week interval. The Pearson's correlation between the first and the second administration of the SO-OCS was .82, indicating adequate temporal stability. At the first administration the total score ranged from 0 to $29(M=2.83 ; S D=4.99)$ and at the retest it ranged from 0 to $27(M=2.35 ; S D=4.66)$; these scores did not significantly differ $(t(99)=1.65, p=.10)$, supporting the test-retest reliability of the scale in non-clinical samples.

\section{Construct validity}

It was predicted that the SO-OCS total score would be more strongly correlated with the OCI-R obsessing subscale (convergent measure) than with other subscales of the OCI-R and with other considered measures (discriminant measures). Pearson's correlations between SO-OCS scores and scores on other measures were calculated both in the NCP group and in the whole OCD group $(n=103)$ and subgroups. As showed in Table 4, the convergent correlation was $.30(p<.01)$ in the NCP group, $.46(p<.05)$ in the SO-OCD group, and $.25(p<.05)$ in the NSO-OCD group. Discriminant correlations ranged from .10 to .20 in the NCP group, from .13 to .31 in the SO-OCD group, and from -.35 to .07 in the NSO-OCD group. As expected, SO-OCS scores were significantly more strongly correlated with subscales that assess specifically obsessional OCD symptoms than with subscales that assess other OCD symptoms, depression, anxiety and worry; $Z_{\text {contrast }}$ tests showed significant differences between convergent and discriminant measures in all groups (NCP: $z=5.51, p<.001$; SO-OCD: $z=2.15, p<.05$; NSO-OCD: $z=2.96, p<.05$ ). These results suggest that the scale has adequate construct validity.

\section{Criterion validity}

The GLM analysis showed that, once controlling for the demographic variables in Table 2, group membership had a significant effect on SO-OCS score $\left(F(2,381)=2077.28, p<.001, \eta^{2}=\right.$ 
.77). Games-Howell post-hoc analyses revealed that scores of SO-OCD group on the SO-OCS were significantly $(p<.001)$ higher than those of either of the other groups, which did not significantly differ from each other. None of the demographic variables had a statistically significant effect.

\section{Diagnostic sensitivity}

In distinguishing individuals with SO-OCD from those with NSO-OCD, the AUC estimate for the SO-OCS total score approached 1.00. In distinguishing individuals with SO-OCD from NCP, the AUC estimate again approached 1.00. These data indicate that the SO-OCS score discriminates individuals with SO-OCD almost perfectly both from non-clinical individuals and from patients with other presentations of $\mathrm{OCD}$, as also suggested by the very large mean differences in scale scores (see Table 3). Next, we examined the accuracy of different SO-OCS cut-off scores in correctly classifying patients as having a primary diagnosis of SO-OCD or a different presentation of obsessive-compulsive disorder (NSO-OCD). We evaluated diagnostic accuracy by calculating the sensitivity and specificity of various SO-OCS total scores. A cut-off score of 21 provided the best balance between sensitivity (.981) and specificity (.997) in distinguishing SO-OCD patients from those with NSO-OCD. Similar analyses revealed that a cut-off score of 18 provided the best balance between sensitivity (.998) and specificity (.999) in distinguishing SO-OCD patients from non-clinical participants.

\section{Discussion}

Sexual Orientation obsessions are an important, yet to-date understudied symptom dimension of OCD. One factor contributing to this dearth of research is the lack of tools for assessing SO-OCD - while other measurement tools exist, they are either too long for routine use or they do not specifically assess SO-OCD. As such, the present study aimed to develop a measure the Sexual Orientation Obsessive-Compulsive Scale - and to evaluate its psychometric properties, using both a large community sample and OCD samples with and without sexual orientation symptoms. The newly developed scale consists of both obsessions and compulsive actions related to SO-OCD, including doubt and self-monitoring regarding one's sexual orientation, checking 
whether one is aroused by same or opposite sex people, and self-analysis regarding one's sexual orientation. Generally, our analyses found a single factor structure and evidence for convergent and divergent validity across all samples, suggesting that the measure is appropriate for its intended use.

Specifically, exploratory factor analyses supported a one-factor measurement model of the SO-OCS items in all the recruited samples (community samples and clinical samples with and without SO-OCD). While confirmatory analyses could not be performed in the clinical sample due to the low sample size, when carried out in the non-clinical samples they convincingly supported a unidimensional measurement model. The SO-OCS showed very good reliability, both via internal consistency indices in the various samples, and when examining temporal stability over a 4-week period in a sub-sample of the non-clinical participants. As such, the measure appears a stable and reliable measure of symptoms, at least in the general population.

This study also provided evidence for the construct validity of the SO-OCS, since its total score was more strongly correlated with the convergent measure of the OCI-R obsessing subscale than with other subscales of the OCI-R and with other discriminant measures. However, correlations with convergent measures were only of moderate strength. The size of this relationship likely reflects that the 3-item OCI obsessing scale is quite general - as such, while it may pick up individuals with sexual obsessions, it would also assess other obsessive content that is not sexualorientation related. This is consistent with the pattern whereby the highest correlation $(r=.46)$ was in the SO-OCD group (as their predominant obsessive symptoms were sexual-orientation related), and lowest in the OCD symptom without predominant sexual-orientation obsessions (as the OCI obsessions scale was likely picking up on other general obsessions). Equally, the SO-OCS did correlate with checking compulsions in the sexual orientation OCD group, but correlations were in fact negative in the non-sexual-orientation OCD group. Again, it is likely that the general 3-item checking scale is picking up on sexual-orientation checking behaviours in the SO-OCD group, but related to other behaviours in those without prominent checking behaviours. The reason this correlation was negative in N-SO-OCD group is not clear; this may be a spurious association due to 
the presence of some outliers or other quirks of the N-SO-OCD group, particularly when considering that $76 \%$ of the participants in NSO-OCD group scored zero at the SO-OCS. However it is possible that within these participants, who have clinical levels of $\mathrm{OCD}$, those with extreme levels of clinical checking are so preoccupied with their physical safety-related checking that it is to the exclusion of compulsions about sexual orientation; i.e., if one is checking for safety at clinically significant levels they simply do not have the time or motivation to check their sexual orientation in the presence of their partner (who may also be checking for them). It should also be considered that that two items out of three from the OCI-R specifically tap physical checking (doors/windows/drawers; taps/light switches, etc.), with only one (I check things more often than necessary) likely to overlap with SO-OCD symptoms. Future studies may aim to explore the convergent association between our SO-OCS scale and the recent adaption of the Dimensional Obsessive-Compulsive Scale (DOCS) that assesses Sexually Intrusive Thoughts as an added dimension (Wetterneck et al., 2015), as well as the longer SO-OCD scale developed by Williams and colleagues (2014). Unfortunately, these scales were not published when the present study was being designed.

Curiously, the other OCD symptom dimension that was significantly correlated with the SOOCS was that which assessed washing symptoms. This result perhaps suggests that the SO-OCD symptoms might lead to feelings of mental contamination. Mental contamination is a psychological state whereby the individual feels an internalized emotional sense of being dirty, for instance, because they have simply imagined certain events (Rachman, 2004). Such feelings are generally evoked by events the individual perceives as wrong, inappropriate or personally immoral (Elliott \& Radomsky, 2009). For example, Fairbrother, Newth, and Rachman (2005) reported an experiment where non-clinical individuals felt a need to wash after imagining a non-consensual kiss at a party, but not after imagining a consensual kiss. While individuals with SO-OCD do not necessarily see acts against their sexual orientation as being immoral, they certainly contrast with their own personal belief system. Future studies examining the SO-OCS against the recently developed 
mental contamination scale (Melli, Carraresi, Stopani, Radomsky, \& Bulli, 2015; Radomsky, Rachman, Shafran, Coughtrey, \& Barber, 2014) would shed light on this relationship. Moreover, it would be interesting to explore the relationship between the SO-OCS and the Fear of Self-

Questionnaire (FSQ; Aardema et al., 2014). This follows research by Melli, Aardema and Moulding (2015) which found that fear-of-self concerns - fear regarding who the person might be or might become - are unique, major predictors of unacceptable/taboo thoughts in clinical samples with OCD, independent of negative mood states and obsessive beliefs.

We also examined the ability of the SO-OCS to discriminate individuals with SO-OCS from other known groups - those without OCD and those with non-sexual-orientation OCD as a primary complaint. The SO-OCS was clearly able to discriminate sexual-orientation OCD from both groups, although a different cut-off score was required to clearly discriminate between the OCD groups (21) compared to the SO-OCD vs. NCP groups (18). It is likely that a higher score was required when comparing the two OCD groups, because even individuals with identified non-sexual-orientation OCD have generally higher OCD symptom levels across other domains. These results supported the excellent criterion validity of the scale, and suggest it could play a role in screening for SO-OCD. Future research is needed to follow-up on this, including interviewing those who score higher than the cut-off in community groups to determine the positive predictive value within that scenario. Furthermore, it would be important to test whether the scale could effectively discriminate between individuals who struggle with accepting or understanding their sexual orientation (and therefore respond to such concerns with fear or anger) and individuals with SO-OCD, as this is a frequently discussed obstacle in clinical care.

This study has some limitations that should be kept in mind. First, the scale was developed and tested in Italian rather than in English. The English translation of the SO-OCS was carried out through a mixed forward- and back-translation procedure. Two of the authors and one bilingual Italian-English psychologist independently translated the items into English. After consensus among translators was achieved, another Italian-English researcher blind to the original version 
translated this preliminary version back into Italian. Discrepancies emerging from this backtranslation were discussed in order to refine the English version. We believe the scale will prove appropriate also in English settings, particularly given that we have found in multiple previous studies of OCD and more generally that psychopathology scales translate across Italian-English settings without significant alterations to their structure or content (e.g., Bottesi et al., 2015; Melli, Chiorri, et al., 2015; Melli, Carraresi, Stopani, Radomsky, \& Bulli, 2014), along with evidence (Williams et al., 2014) and experience that SO-OCD symptoms are common in English settings. However, future studies are required to firmly establish the psychometric properties of any English language version, along with determining appropriate cut-off points. Second, while the scale has a clear pattern of convergent and divergent validity, and known-group validity was good, at the time of devising this study other measures of SO-OCD were not available: hence, additional studies utilizing the other recently developed convergent measures (particularly those by Wetterneck et al., 2015; Williams et al., 2014) would strengthen the claims made in this article. Third, although we provide preliminary evidence of temporal stability of the SO-OCS in a non-clinical sample, this study did not test sensitivity to change in treated patients nor test-retest reliability in non-treated patients. Further research is warranted to establish whether it can reliably be used as a measure of therapeutic change and/or over longer periods of time. Fourth, all the participants in clinical samples were self-selected: therefore, the samples may not have been representative of the relevant clinical populations. In particular, gender ratios in NSO-OCD group (71\% males) and SO-OCD group ( $80 \%$ males) were not representative of OCD in general. The high ratio of men to woman in the clinical groups and the relatively small sample size of the NSO-OCD group compared to that of the SO-OCD group - considering the ratio of SO-OCD to OCD in general - may limit the generalizability of the findings. Finally, as already reported, given the predominance of homosexual concerns within heterosexual populations with SO-OCD, the first version of the scale has been developed focusing on these concerns. We also have adapted the scale content to reflect 
heterosexual sexual-orientation related concerns in L.G.B.T. populations (SO-OCS-V2; available on request). However, future studies would be required to test the psychometrics of such a version. In conclusion, while considering the limitations described above, this study provided evidence that our newly developed scale that aimed to measure sexual-orientation obsessions - the SO-OCS - had sound psychometric properties and could be confidently employed in clinical and research settings in which sexual orientation-related symptoms or concerns are of interest. We believe that the SO-OCS is potentially an important measure for use in comprehensive assessment batteries, in addition to scales assessing more traditional OCD symptomatology (e.g., contamination, checking, etc.), and semi-structured interviews. The SO-OCS is quick to administer and score, and is available for clinical and research use without cost. 


\section{References}

Aardema, F., Moulding, R., Radomsky, A. S., Doron, G., Allamby, J., \& Souki, E. (2013). Fear of self and obsessionality: Development and validation of the Fear of Self Questionnaire. Journal of Obsessive-Compulsive and Related Disorders, 2, 306-315.

American Psychiatric Association (2013). Diagnostic and statistical manual of mental disorders, (DSM-5®). Washington D.C.: American Psychiatric Publishing.

American Psychological Association (2010). Ethical principles of psychologists and code of conduct. http://www.apa.org/ethics/code/principles.pdf

Beck, A. T., \& Steer, R. A. (1990). Manual for the Beck Anxiety Inventory. San Antonio, TX: Psychological Corporation.

Beck, A. T., Steer, R. A., \& Brown, G. K. (1996). Manual for the beck depression inventory-II. San Antonio, TX: Psychological Corporation.

Bloch, M. H., Landeros-Weisenberger, A., Rosario, M. C., Pittenger, C., \& Leckman, J. F. (2008). Meta-analysis of the symptom structure of obsessive-compulsive disorder. The American Journal of Psychiatry, 165, 1532-1542.

Bottesi, M., Ghisi, M., Altoè, G., Conforti, E., Melli, G., \& Sica, C. (2015). The Italian version of the Depression Anxiety Stress Scales-21: Factor structure and psychometric properties on community and clinical samples. Comprehensive Psychiatry, 60, 170-181.

Brown, T. A., Barlow, D. H., \& Di Nardo, P. A. (1994). Anxiety Disorders Interview Schedule Adult Version: Client interview schedule. Oxford: Oxford University Press.

Buja, A., \& Eyuboglu, N. (1992). Remarks on parallel analysis. Multivariate behavioral research, 27, 509-540.

Burns, G. L., Keortge, S. G., Formea, G. M., \& Sternberger, L. G. (1996). Revision of the Padua Inventory of obsessive compulsive disorder symptoms: distinctions between worry, obsessions, and compulsions. Behaviour Research and Therapy, 34, 163-173. 
Cathey, A. J., \& Wetterneck, C. T. (2013). Stigma and disclosure of intrusive thoughts about sexual themes. Journal of Obsessive-Compulsive and Related Disorders, 2, 439-443.

de Winter, J. C. F., Dodou, D., \& Wieringa, P. A. (2009). Exploratory factor analysis with small sample sizes. Multivariate behavioral research, 44, 147-181.

Elliott, C. M., \& Radomsky, A. S. (2009). Analyses of mental contamination: Part I, experimental manipulations of morality. Behaviour Research and Therapy, 47, 995-1003.

Fairbrother, N., Newth, S. J., \& Rachman, S. (2005). Mental pollution: Feelings of dirtiness without physical contact. Behaviour Research and Therapy, 43, 121-130.

Foa, E. B., Huppert, J. D., Leiberg, S., Langner, R., Kichic, R., Hajcak, G., \& Salkovskis, P. M. (2002). The Obsessive-Compulsive Inventory: development and validation of a short version. Psychological Assessment, 14, 485.

Furr, M. (2011). Scale construction and psychometrics for social and personality psychology. Los Angeles: SAGE.

Goldberg, R. L. (1984). Heterosexual panic. The American journal of psychoanalysis, 44, 209-211.

Gordon, W. M. (2002). Sexual obsessions and OCD. Sexual and Relationship Therapy, 17, 343-354.

Kaiser, H. F. (1974). An index of factorial simplicity. Psychometrika, 39, 31-36.

Kline, P. (1993). The Handbook of Psychological Testing. London: Routledge.

Longman, R. S., Cota, A. A., Holden, R. R., \& Fekken, G. C. (1989). A regression equation for the parallel analysis criterion in principal components analysis: Mean and 95th percentile eigenvalues. Multivariate behavioral research, 24, 59-69.

Lorenzo-Seva, U., \& Ferrando, P. J. (2006). FACTOR: A computer program to fit the exploratory factor analysis model. Behavior Research Methods, 38, 88-91.

Marsh, H. W., Hau, K.-T., \& Wen, Z. (2004). In search of golden rules: Comment on hypothesistesting approaches to setting cutoff values for fit indexes and dangers in overgeneralizing Hu and Bentler's (1999) findings. Structural Equation Modeling, 11, 320-341. 
Melli, G., Aardema, F., \& Moulding, R. (2015). Fear of Self and Unacceptable Thoughts in Obsessive-Compulsive Disorder. Clinical Psychology and Psychotherapy. DOI: 10.1002/cpp.1950 Melli, G., Avallone, E., Moulding, R., Pinto, A., Micheli, E., \& Carraresi, C. (2015). Validation of the Italian version of the Yale-Brown Obsessive Compulsive Scale-Second Edition (Y-BOCS-II) in a clinical sample. Comprehensive Psychiatry, 60, 86-92.

Melli, G., Carraresi, C., Stopani, E., Radomsky, A., \& Bulli, F. (2015). Factor structure and temporal stability of the Vancouver Obsessional Compulsive Inventory-Mental Contamination Scale (VOCIMC) and psychometric properties of its Italian version. Comprehensive Psychiatry, 58, 198-204.

Melli, G., Chiorri, C., Bulli, F., Carraresi, C., Stopani, E., \& Abramowitz, A. (2015). Factor congruence and psychometric properties of the Italian version of the Dimensional ObsessiveCompulsive Scale (DOCS) across non-clinical and clinical samples. Journal of Psychopathology and Behavioral Assessment, 37, 329-339.

Metz, C. E. (1978). Basic principles of ROC analysis. Paper presented at the Seminars in Nuclear Medicine.

Meyer, T. J., Miller, M. L., Metzger, R. L., \& Borkovec, T. D. (1990). Development and validation of the Penn State Worry Questionnaire. Behaviour Research and Therapy, 28, 487-495.

Morani, S., Pricci, D., \& Sanavio, E. (1999). Penn State Worry Questionnaire e Worry Domains Questionnaire. Presentazione delle versioni italiane ed analisi della fedeltà. Psicoterapia cognitiva e comportamentale, 5, 195-209.

Moulding, R., Aardema, F., \& O'Connor, K. P. (2014). Repugnant obsessions: A review of the phenomenology, theoretical models, and treatment of sexual and aggressive obsessional themes in OCD. Journal of Obsessive-Compulsive and Related Disorders, 3, 161-168.

Murray, C. J. L., \& Lopez, A. D. (1996). The global burden of disease and injury series, volume 1: a comprehensive assessment of mortality and disability from diseases, injuries, and risk factors in 1990 and projected to 2020. World Health Organisation. 
Muthén, B., Du Toit, S. H., \& Spisic, D. (1997). Robust inference using weighted least squares and quadratic estimating equations in latent variable modeling with categorical and continuous outcomes. Psychometrika, 75, 1-45.

Muthén, B., \& Kaplan, D. (1985). A comparison of some methodologies for the factor analysis of nonnormal Likert variables. British Journal of Mathematical and Statistical Psychology, 38, 171-189.

Muthén, L. K., \& Muthén, B. O. (2002). How to use a Monte Carlo study to decide on sample size and determine power. Structural Equation Modeling, 9, 599-620.

Nedeljkovic, M., Moulding, R., Foroughi, E., Kyrios, M., \& Doron, G. (2012). Cultural issues in understanding and treating obsessive compulsive and spectrum disorders. In G. Steketee (Ed.), Oxford handbook of obsessive compulsive and spectrum disorders (pp. 496-520). Oxford: Oxford University Press.

Pinto, A., Greenberg, B. D., Grados, M. A., Bienvenu, O. J., Samuels, J. F., Murphy, D. L., . . Shugart, Y. Y. (2008). Further development of YBOCS dimensions in the OCD Collaborative Genetics study: symptoms vs. categories. Psychiatry Research, 160, 83-93.

Rachman, S. (1971). Obsessional ruminations. Behaviour Research and Therapy, 9, 229-235.

Rachman, S. J. (2004). Fear of contamination. Behaviour Research and Therapy, 42, 1227-1255.

Radomsky, A., Rachman, S. J., Shafran, R., Coughtrey, A. E., \& Barber, K. C. (2014). The nature and assessment of mental contamination: a psychometric analysis. Journal of Obsessive Compulsive and Related Disorders, 3, 181-187.

Samuels, J. F., Riddle, M. A., Greenberg, B. D., Fyer, A. J., McCracken, J. T., Rauch, S. L., . . . Knowles, J. A. (2006). The OCD collaborative genetics study: methods and sample description. American Journal of Medical Genetics Part B: Neuropsychiatric Genetics, 141, 201-207.

Sica, C., Coradeschi, D., Ghisi, M., \& Sanavio, E. (2006). Beck Anxiety Inventory. Manuale. Firenze: Organizzazioni Speciali. 
Sica, C., \& Ghisi, M. (2007). The Italian Versions of the Beck Anxiety Inventory and the Beck Depression Inventory-II: psychometric properties and discriminant power. In M. A. Lange (Ed.), Leading-edge psychological tests and testing (pp. 27-50). New York: Nova Science Publishers.

Sica, C., Ghisi, M., Altoè, G., Chiri, L. R., Franceschini, S., Coradeschi, D., \& Melli, G. (2009). The Italian version of the Obsessive Compulsive Inventory: Its psychometric properties on community and clinical samples. Journal of Anxiety Disorders, 23, 204-211.

Storch, E. A., Rasmussen, S. A., Price, L. H., Larson, M. J., Murphy, T. K., \& Goodman, W. K. (2010). Development and psychometric evaluation of the Yale-Brown Obsessive-Compulsive Scale-Second Edition. Psychological Assessment, 22, 223-232.

Swets, J. A. (1996). Signal detection theory and ROC analysis in psychology and diagnostics: Collected papers. New Jersey: Lawrence Erlbaum.

Szymanski, D. M., Kashubeck-West, S., \& Meyer, J. (2008). Internalized heterosexism measurement, psychosocial correlates, and research directions. The Counseling Psychologist, 36, 525-574.

Timmerman, M. E., \& Lorenzo-Seva, U. (2011). Dimensionality assessment of ordered polytomous items with parallel analysis. Psychological Methods, 16, 209.

Velicer, W. F. (1976). Determining the number of components from the matrix of partial correlations. Psychometrika, 41, 321-327.

Weissman, M., Bland, R., Canino, G., Greenwald, S., Hwu, H., Lee, C., . . Wickramaratne, P. (1994). The Cross National Collaborative Group. The cross national epidemiology of obsessive compulsive disorder. Journal of Clinical Psychiatry, 55(suppl. 3), 5-10.

Weston, D., \& Rosenthal, R. (2003). Quantifying construct validity: Two simple measures. Journal of Personality and Social Psychology, 84, 608-618.

Wetterneck, C. T., Siev, J., Adams, T. G., Slimowicz, J. C., \& Smith, A. H. (2015). Assessing sexually intrusive thoughts: Parsing unacceptable thoughts on the Dimensional Obsessive-Compulsive Scale. Behavior Therapy, 46, 544-556. 
Williams, M. T. (2008). Homosexuality anxiety: A misunderstood form of OCD. In L. V. Sebeki (Ed.), Leading-edge Health Education Issues (pp. 195-205). New York: Nova Science.

Williams, M. T., Crozier, M., \& Powers, M. (2011). Treatment of sexual-orientation obsessions in obsessive-compulsive disorder using exposure and ritual prevention. Clinical Case Studies, 10, 5366.

Williams, M. T., \& Farris, S. G. (2011). Sexual orientation obsessions in obsessive-compulsive disorder: Prevalence and correlates. Psychiatry Research, 187, 156-159.

Williams, M. T., Tellawi, G., Davis, D. M., \& Slimowicz, J. C. (2015). Assessment and Treatment of Sexual Orientation Obsessions in Obsessive-Compulsive Disorder. Australian Clinical Psychology, $1,12-18$.

Williams, M. T., Wetterneck, C., Tellawi, G., \& Duque, G. (2014). Domains of distress among people with sexual orientation obsessions. Archives of sexual behavior, 44, 783-789. 
Assessment of sexual orientation-related OCD 29

\section{Footnotes}

1. Please note that we use "gay" for same-sex sexual-orientation regardless of the individual's gender. We also use this term where possible in preference to "homosexual" or "homosexuality" given the historically negative connotations associated with these terms, outside of the discussion of "Homosexual OCD" which is a term that has been utilized in the literature. 
Table 1

Item descriptive statistics, item analyses and factor loadings from the exploratory factor analysis of the SO-OCS in Study 1 ( $\mathrm{n}=732$ ).

\begin{tabular}{|c|c|c|c|c|c|c|c|c|c|c|}
\hline Item & & $M$ & $S D$ & Range & SK & KU & $r_{\text {it }}$ & $\alpha w / o$ & Loading & Communality \\
\hline 1. & I often wonder what my sexual orientation is. & .18 & .49 & $0-4$ & 3.40 & 13.88 & .58 & .88 & .79 & .62 \\
\hline 2. & I constantly seek evidence that proves my sexual orientation. & .08 & .31 & $0-3$ & 4.76 & 25.91 & .64 & .87 & .87 & .76 \\
\hline 3. & $\begin{array}{l}\text { I often worry about suddenly discovering that I am } \\
\text { homosexual. }\end{array}$ & .12 & .41 & $0-4$ & 4.26 & 22.03 & .62 & .88 & .83 & .69 \\
\hline 4. & $\begin{array}{l}\text { I monitor the sensations that I feel when I am face-to-face } \\
\text { with people in order to understand which is my sexual } \\
\text { orientation. }\end{array}$ & .12 & .42 & $0-4$ & 4.77 & 29.83 & .51 & .88 & .80 & .64 \\
\hline 5. & $\begin{array}{l}\text { I'm afraid to find out that my real sexual orientation is } \\
\text { homosexual. }\end{array}$ & .06 & .30 & $0-4$ & 6.68 & 61.29 & .58 & .88 & .84 & .71 \\
\hline 6. & $\begin{array}{l}\text { I feel the need to repeatedly check if I am attracted to men or } \\
\text { women. }\end{array}$ & .13 & .44 & $0-4$ & 4.30 & 22.28 & .55 & .88 & .79 & .62 \\
\hline 7. & $\begin{array}{l}\text { I worry excessively about not being adequately attracted to } \\
\text { opposite-sex people and that this is evidence that I am } \\
\text { homosexual. }\end{array}$ & .05 & .25 & $0-3$ & 6.13 & 45.73 & .54 & .88 & .84 & .71 \\
\hline 8. & $\begin{array}{l}\text { I often analyze my past and my life history seeking evidence } \\
\text { of my heterosexual orientation. }\end{array}$ & .10 & .42 & $0-4$ & 5.85 & 41.10 & .46 & .88 & .79 & .62 \\
\hline 9. & $\begin{array}{l}\text { I am constantly annoyed by the doubt that my sexual } \\
\text { orientation could differ from the one that I have always } \\
\text { believed. }\end{array}$ & .05 & .23 & $0-3$ & 4.94 & 25.84 & .59 & .88 & .86 & .74 \\
\hline 10. & $\begin{array}{l}\text { I often check that I am still attracted to opposite-sex people to } \\
\text { reassure myself that I am heterosexual. }\end{array}$ & .08 & .32 & $0-3$ & 4.52 & 23.42 & .60 & .88 & .83 & .69 \\
\hline 11. & $\begin{array}{l}\text { I am obsessed with doubts about the possibility that I could be } \\
\text { homosexual. }\end{array}$ & .08 & .29 & $0-3$ & 3.95 & 16.24 & .66 & .87 & .88 & .77 \\
\hline 12. & $\begin{array}{l}\text { I often check what sensations I feel towards same-sex people, } \\
\text { in order to be sure that I don't feel anything that could reveal } \\
\text { homosexual tendencies. }\end{array}$ & .08 & .30 & $0-3$ & 4.05 & 17.23 & .61 & .88 & .87 & .76 \\
\hline 13. & $\begin{array}{l}\text { I often worry that I could be homosexual and that I am } \\
\text { denying it to myself. }\end{array}$ & .06 & .27 & $0-3$ & 4.54 & 21.92 & .63 & .88 & .87 & .76 \\
\hline 14. & $\begin{array}{l}\text { I check myself, physically and mentally, to ensure that I am } \\
\text { not sexually aroused when I happen to see an attractive same- }\end{array}$ & .07 & .28 & $0-3$ & 5.42 & 38.01 & .57 & .88 & .84 & .71 \\
\hline
\end{tabular}
sex person.

Note: SO-OCS = Sexual Orientation Obsessive-Compulsive Scale; $M=$ Mean; $S D=$ Standard Deviation; $\mathrm{SK}=\mathrm{Skewness} ; \mathrm{KU}=\mathrm{Kurtosis} ; r_{\mathrm{it}}=\mathrm{corrected}$ item-total correlation; $\alpha$ w/o = Cronbach's alpha-if-item-deleted. 
Table 2

Demographic characteristics and descriptive data for the SO-OCS across the groups in Study 2.

\begin{tabular}{llrrrr}
\hline Variable & Category & NCP $(\boldsymbol{n}=\mathbf{2 9 4})$ & SO-OCD $(\boldsymbol{n}=\mathbf{5 2})$ & NSO-OCD $(\boldsymbol{n}=\mathbf{5 1})$ \\
\hline Sex (proportion) & Male & $38 \%$ & $80 \%$ & $71 \%$ \\
& Female & $62 \%$ & $20 \%$ & $29 \%$ \\
Age $(\boldsymbol{M} \pm \boldsymbol{S D})$ & & $35.30 \pm 14.65$ & $25.90 \pm 6.95$ & $34.31 \pm 10.19$ & \\
Years of education & & $14.30 \pm 3.67$ & $13.21 \pm 3.30$ & $14.10 \pm 3.61$ & \\
$(\boldsymbol{M} \pm \boldsymbol{S D})$ & & & & $61 \%$ \\
Marital status & Single & $49 \%$ & $88 \%$ & $31 \%$ \\
& Married & $47 \%$ & $8 \%$ & $8 \%$ \\
& Divorced/widow & $4 \%$ & $4 \%$ & $2 \%$ \\
Occupation & Homemaker & $7 \%$ & $0 \%$ & $35 \%$ \\
& Employee & $21 \%$ & $25 \%$ & $18 \%$ \\
& Professional & $11 \%$ & $40 \%$ & $8 \%$ \\
& Unoccupied & $5 \%$ & $0 \%$ & $0 \%$ \\
& Retired & $7 \%$ & $54 \%$ & $23 \%$ \\
& Student & $35 \%$ & $7 \%$ & $14 \%$ \\
\hline
\end{tabular}

Note: SO-OCS = Sexual Orientation Obsessive-Compulsive Scale; NCP = Non-clinical participants; SO-OCD = Sexual Orientation related ObsessiveCompulsive Disorder; NSO-OCD = Non Sexual Orientation related Obsessive-Compulsive Disorder; $M=$ Mean; $S D=$ Standard deviation. 
Table 3

Distribution and item analyses for the SO-OCS across the groups in Study 2.

\begin{tabular}{|c|c|c|c|}
\hline & $\begin{array}{l}\text { NCP } \\
(n=294)\end{array}$ & SO-OCD $(n=52)$ & NSO-OCD $(n=51)$ \\
\hline SO-OCS total score $(M \pm S D)$ & $1.36 \pm 3.30$ & $46.44 \pm 7.98$ & $1.86 \pm 4.02$ \\
\hline Item skewness (range) & 3.84 to 7.52 & -.65 to -1.83 & 2.17 to 4.89 \\
\hline Item kurtosis (range) & 15.10 to 66.96 & -.71 to 3.24 & 2.83 to 22.83 \\
\hline Cronbach's Alpha & .85 & .90 & .89 \\
\hline Highest alpha-if-item-deleted value & .84 & .90 & .89 \\
\hline Mean inter-item correlation & .33 & .41 & .43 \\
\hline Corrected item-total correlation (range) & .35 to. 66 & .42 to. 81 & .32 to. 81 \\
\hline
\end{tabular}

Note: SO-OCS = Sexual Orientation Obsessive-Compulsive Scale; NCP = Non-clinical Participants; SO-OCD = Sexual Orientation related ObsessiveCompulsive Disorder; NSO-OCD = Non Sexual Orientation related Obsessive-Compulsive Disorder. 
Table 4

Construct validity of the SO-OCS. Pearson's correlations between SO-OCS scores and scores on other measures by group in Study 2.

\begin{tabular}{llllllllll}
\hline Group & $\begin{array}{l}\text { OCI-R } \\
\text { Obsessing }\end{array}$ & $\begin{array}{l}\text { OCI-R } \\
\text { Hoarding }\end{array}$ & $\begin{array}{l}\text { OCI-R } \\
\text { Checking }\end{array}$ & $\begin{array}{l}\text { OCI-R } \\
\text { Ordering }\end{array}$ & $\begin{array}{l}\text { OCI-R } \\
\text { Mental } \\
\text { neutralizing }\end{array}$ & $\begin{array}{l}\text { OCI-R } \\
\text { Washing }\end{array}$ & PSWQ & BDI-II & BAI \\
\hline NCP $(n=294)$ & $.30^{* *}$ & .11 & $.12^{*}$ & .10 & $.17^{* *}$ & $.20^{* *}$ & $.12^{*}$ & $.12^{*}$ & $.18^{* *}$ \\
SO-OCD $(n=52)$ & $.46^{*}$ & .13 & $.31^{*}$ & .22 & .18 & $.29^{*}$ & .20 & .24 & .17 \\
NSO-OCD $(n=51)$ & $.25^{*}$ & .07 & $-.35^{*}$ & $-.27^{*}$ & -.05 & -.23 & -.03 & -.06 & -.12 \\
\hline
\end{tabular}

$* p<.05-* * p<.01$

Note: SO-OCS = Sexual Orientation Obsessive-Compulsive Scale; NCP = Non-clinical Participants; SO-OCD = Sexual Orientation related ObsessiveCompulsive Disorder; NSO-OCD = Non Sexual Orientation related Obsessive-Compulsive Disorder; OCI-R = Obsessive-Compulsive Inventory-Revised; $\mathrm{PSWQ}=$ Penn State Worry Questionnaire; BDI-II = Beck Depression Inventory-II; BAI = Beck Anxiety Inventory. 